\title{
Real-Time Data Processing using Python in DigitalMicrograph
}

\author{
Ben Miller ${ }^{*}$, Stephen Mick ${ }^{1}$ \\ 1. Gatan Inc. Pleasanton, CA, USA. \\ * Corresponding author: BMiller@gatan.com
}

Processing and visualizing data after a transmission electron microscopy (TEM) experiment is an essential step toward generating scientific conclusions. While detailed processing and visualization may take place long after data collection, it can be beneficial to perform initial processing and visualization rapidly during a session on the microscope. This initial analysis enables users to determine whether good data is being collected, the experimental conditions need to be modified, or additional data collection is needed. Enabling the user to do this assessment while the experiment is still in progress can save hours or days of preparation for follow-up experiments if deficiencies with the data are not discovered until the TEM session is over. This time savings is especially true for in-situ experiments where a researcher may only have one chance to capture irreversible dynamics in any given TEM sample. With in situ experiments, it is critical to analyze what is occurring with the sample in real-time since the next experimental step at the microscope is often decided by interpreting the dynamic changes happening in the sample.

Gatan cameras and spectrometers are widely used to collect data during TEM experiments. Gatan's software, Gatan Microscopy Suite ${ }^{\circledR}$ (GMS) also commonly known as DigitalMicrograph ${ }^{\circledR}$ (DM), is used for controlling data acquisition and visualizing the results. GMS has always had scripting capabilities, but the scripting language has been specific to GMS. While this means that it is uniquely tailored to the needs of microscopists, it also has limited the use of open-source libraries within GMS during TEM data collection.

Gatan has now integrated Python scripting into GMS adding the capability to edit and run Python code directly from the scripting interface in GMS. With its own Python API, GMS enables a Python code to interact with all the native Gatan objects such as images, windows, tags, and even cameras and microscopes. Python libraries and packages can also be imported and used, unlocking endless possibilities for processing, visualization, and analysis during data collection.

Figure 1 shows the results of a processing script written in Python for use in GMS. This script monitors the live view window, processing full resolution images live as they are displayed. The FFT is computed for each image, and then a radial profile of the FFT is produced by subtracting a radial average from a radial maximum. Each profile created in this way is added as a single row of an image which thus tracks changes in the FFT as a function of time. This method enables the viewer to easily see changes in the crystallinity of the material in the field of view [1]. Phase transformations, crystallization, or amorphization due to beam damage can all be monitored in real time with this script while the full resolution data is being acquired. The material in Figure 1 is a highly sensitive zeolite sample and the degradation of the crystal structure due to the electron beam is clearly visible toward the end of the observation.

Integration of Python scripting into GMS will enable powerful processing algorithms to be implemented on data during acquisition. As shown in this example, real-time data processing enables immediate visualization of a dynamic reaction as it is in progress. This can be extended to analyze real-time data 
from the large multi-dimensional datasets that the latest generation of cameras and spectrometers can produce. Since Python is a widely adopted scripting language [2], its integration into GMS will make TEM data processing accessible to far more researchers. Perhaps most importantly, as real-time processing is increasingly adopted, machine learning based approaches that are readily implemented with Python libraries can be applied to rapidly answer questions at the microscope that just a few years ago required extensive post-processing [3].

References:

[1] BK Miller et al., Microscopy and Microanalysis 24 (2018), p. 1882.

[2] https://www.tiobe.com/tiobe-index (Accessed Feb, 2019).

[3] The zeolite sample used in this work was kindly provided by Dr. Shery Chang (ASU).
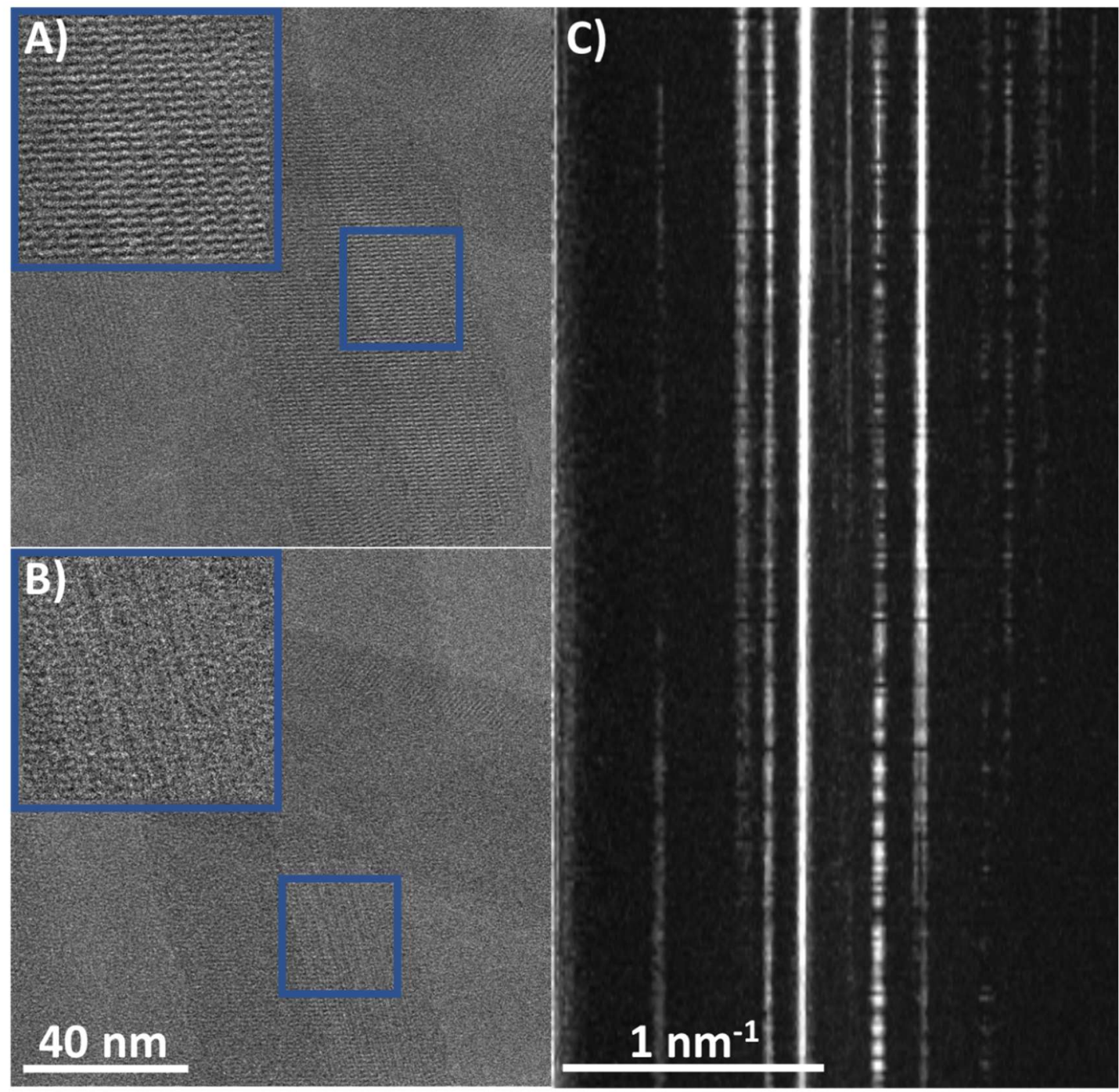

Figure 1. Degradation of a hydroxycancrinite zeolite with $\mathrm{Si} / \mathrm{Al}$ ratio of 1.2 which is highly sensitive to the electron beam. A) Image at the start of observation, with an inset showing that the sample is highly ordered. B) Image after 35 seconds, showing disruption of the crystal structure. C) Series of radial profiles generated live by a Python script acting on the live view window. 